\title{
PHOTON- AND PHONON-ASSISTED TRANSPORT OF ELECTRONS IN RESONANT TUNNELING STRUCTURES
}

\author{
M. V. Tkach, Ju. O. Seti, O. M. Voitsekhivska, O. Yu. Pytiuk \\ Chernivtsi National University, 2, Kotsiubynsky St., UA-58012, Chernivtsi, \\ e-mail: ktf@chnu.edu.ua
}

(Received 14 January, 2017)

\begin{abstract}
The quantum mechanical theory of photon-and phonon assisted tunneling of electrons through the open multilayered nanostructures being the cascades of quantum cascade detector and quantum cascade laser is developed. The theory of electronic dynamic conductivity and spectral parameters (resonance energies and widths) of their quasi-stationary states is established within the effective mass approximation and models of rectangular and triangular potential profiles of resonant tunneling structure, respectively.

It is shown that an open model of multilayered resonant tunneling structure, comparing with the closed one, adequately describes the physical processes in cascades and their elements (active regions, extractors, injectors) and, with respect to the experimental data, gives an opportunity to calculate and reveal their optimal geometrical design without any fitting parameters.
\end{abstract}

Key words: resonant tunneling structure, quantum cascade detector, quantum cascade laser.

PACS number(s): 78.67.De, 63.20.Kr, 72.10.Di, 85.30.Mn, 81.07.St

\section{INTRODUCTION}

It is well known that a long time has passed since the proposed theoretical ideas and principles of the quantum cascade laser (QCL) [1,2] and quantum cascade detector (QCD) [3,4] up untill their practical utilization $[5,6]$ and [7]. It was caused by the technological difficulties while producing the equal cascades of quantum wells and barriers and lack of theory clearly describing the physical phenomena and processes in the multilayered resonant tunneling structure (RTS).

After the first successful experimental papers, the interest of researches sharply increased because these nanodevices had a unique utilization perspectives in science, industry, medicine, military defense and elsewhere [8,9]. They are functioning in the actual infra red range of electromagnetic field frequencies and have other unique characteristics, operating at the inter subband transitions.

The first experimental low temperature QCL [10] has been operated on the injector and QCDs [7,8] on the extractor principles. The new technological abilities and a deeper understanding of physical properties brought to the successfully functioning of QCL $[11,12]$ and QCD $[13,14]$ at high (room) temperatures. The technology of the production of nano-devices has been rapidly developing, which fact caused the appearence of injectorless QCL [15,16] and bi-functional QCL and QCD [17].

The modern QCL and QCD are operating in the whole infrared range. The results of experimental investigations of these nano-devices, operating in the middle and far range, are generalized and analyzed in detail in reviews [18-20] and the near region is discussed in Ref. [21].

The main elements of QCL or QCD are the cascades of RTS, where the electromagnetic field is radiated or absorbed due to the electronic current. The main problem of the theory of electronic transport through the RTS is to reveal the physical models and mathematical approaches which adequately describe physical processes and give an opportunity to obtain the optimal design of cascades and elements of nano-devices. Different approaches are now used in order to calculate the design of QCL and QCD cascades: the methods of the solution of quantum mechanical Schrodinger equation [22, 23], the method of non-equilibrium Green's functions [24-26], multivalley Monte-Carlo simulations [27-29], the density matrix method [30,31] and so on.

The theoretical methods of the calculation of spectral parameters of RTS, being the elements of nano-devices, are presented in the book [32] and reviews [33,34]. The last achievements of the theory concerning the photonassisted transport in multilayered RTS are presented in reviews $[35,36]$ and original papers $[37,38]$.

\section{MAIN PRINCIPLES OF QUANTUM CASCADE LASER AND QUANTUM CASCADE DETECTOR OPERATING IN THE MIDDLE INFRARED RANGE}

The majority of theoretical approaches are based, explicitly or implicitly, on the model of closed RTS with infinitely wide outer potential barriers. According to quantum mechanics, in such closed system, without taking into account the dissipative processes, the quasi-particles are in the stationary states and, thus, their current is absent. However, the situation is paradoxical - the closed models of RTS do not explain the absent current, but the theories developed at their base give an opportunity to calculate the designs of QCD and QCL cascades which rather well correlate with experimental data. So, the problem is to develop an adequate theory which would simultaneously enable us to predict the optimal design of the multilayered RTS. 
The typical principles of functioning of the QCL injector and QCD extractor, used untill now, are presented in Figs. 1a,b, respectively. The RTS driven by the constant electric field with intensity $F$ (Fig. 1,a), being the QCL cascade, contains an active region. The electronic current impinges into it getting into the third quasistationary state (QSS) from the injector of the previous cascade. Due to the quantum transition accompanied by the radiation of electromagnetic field, the electrons from the third state get into the second one. From it, within the radiationless (phonon) mechanism they transit into the ground state of this active region. Further, from the second and ground states, due to tunneling through the injector of a cascade, the electrons get into the third QSS of the next cascade. The conformed operation of all cascades leads to the QCL functioning.
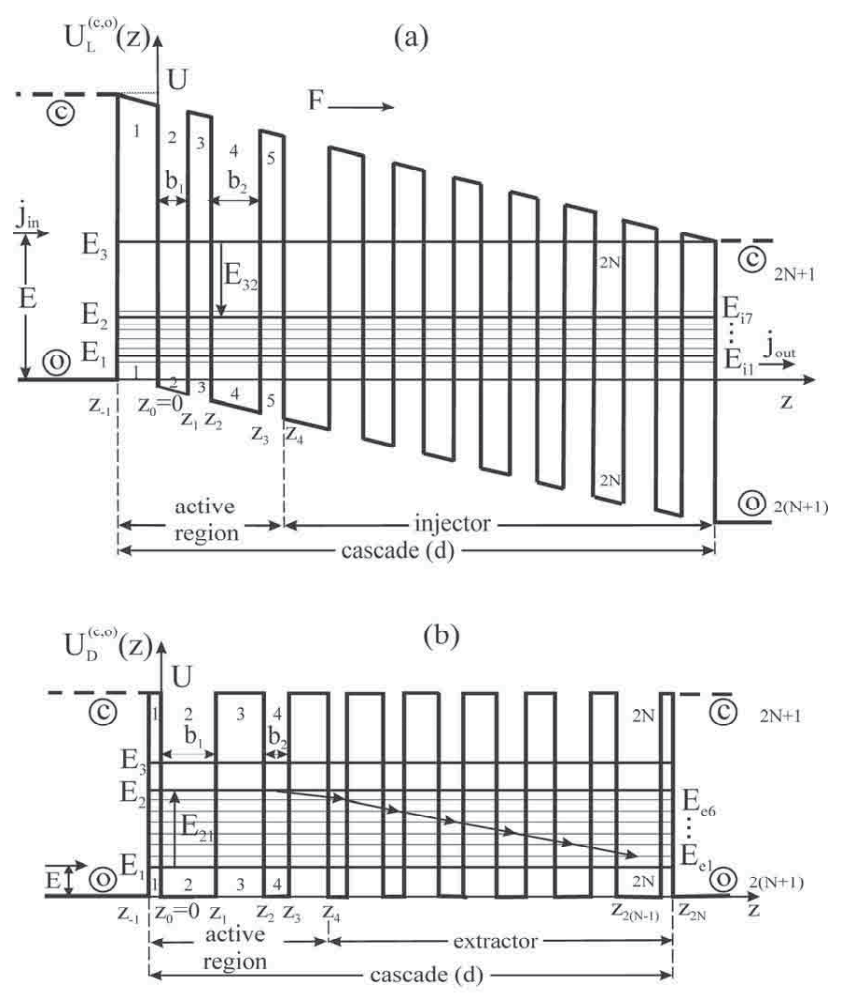

Fig. 1. Potential profile of QCL (a) and QCD (b) in closed (- - -) and open ( - ) models.

The multilayered RTS, being the QCD cascade (Fig. $1, \mathrm{~b})$, contains the active region where the electrons absorbing the electromagnetic field energy transit from the ground QSS into the second one. Then, tunneling through the extractor within the so-called "phonon ladder" and losing part of energy due to a radiationless creation of phonons, the electrons relax and get into the ground QSS of the next cascade.

In this paper, we present the theory of photon- and phonon-assisted tunneling of electrons through the multilayered RTS in an open model (with the nano-size out- er barriers), being the cascades of QCD or QCL. In this model the electronic states are the quasi-stationary ones with the finite life times. Thus, the electronic current is observed. It is clear that the open model of RTS is more adequate to the real cascades of nano-devices and the abovementioned paradox is explained. The results of calculated cascades designs well coincide with the experiment $[39,40]$.

\section{MAIN BACKGROUND OF THE THEORY OF PHOTON-ASSISTED TRANSPORT IN THE OPEN MODEL OF THE PLANE MULTILAYERED RTS AND QUANTUM TRANSITIONS IN THE CLOSED MODEL}

The theory of electron quantum transitions in the closed (c) model or photon assisted electronic transport through the multilayered RTS in the open (o) model of QCL cascade (L), (Fig. 1,a) and QCD cascade (D), (Fig. 1,b) is developed in a similar way for the sake of compactness. According to the one-dimensional models of RTS $[39,40]$, the Hamiltonians of the electron are written as

$$
H_{\mathrm{L}, \mathrm{D}}^{(\mathrm{c}, \mathrm{o})}(z)=-\frac{\hbar}{2} \frac{\partial}{\partial z} \frac{1}{m(z)} \frac{\partial}{\partial z}+U_{\mathrm{L}, \mathrm{D}}^{(\mathrm{c}, \mathrm{o})}(z) .
$$

Here, the effective mass of the electron

$$
m_{z}= \begin{cases}m_{w}, & \text { in well } \\ m_{b}, & \text { in barrier }\end{cases}
$$

is assumed the same as in the bulk crystals for the wells $\left(m_{w}\right)$ and barriers $\left(m_{b}\right)$.

When the RTS is a separate $N$-well cascade of the laser type driven by the constant electric field with the intensity $\mathbf{F} \| \mathbf{n}_{z}$ its potential profile $U_{L}^{(\mathrm{c}, \mathrm{o})}(z)$ has the form (Fig. 1a)

$U_{L}^{(\mathrm{c}, \mathrm{o})}(z)=U_{D}^{(\mathrm{c}, \mathrm{o})}(z)-e F\{z[\theta(z)-\theta(z-d)]+d \theta(z-d)\}$,

$$
U_{D}^{(\mathrm{c}, \mathrm{o})}(z)= \begin{cases}0, & \text { in well } \\ U, & \text { in barrier }\end{cases}
$$

where $U_{D}^{(\mathrm{c}, \mathrm{o})}(z)$ are the potential profiles for the detectortype closed or open model (Fig. 1,b). The height of the potential barrier $(U)$ is assumed as known and fixed by the magnitudes of affinities and energy gaps of contacting crystals.

The solutions of stationary Schrodinger equations

$$
H_{\mathrm{L}, \mathrm{D}}^{(\mathrm{c}, \mathrm{o})}(z) \Psi_{\mathrm{L}, \mathrm{D}}^{(\mathrm{c}, \mathrm{o})}(z)=E \Psi_{\mathrm{L}, \mathrm{D}}^{(\mathrm{c}, \mathrm{o})}(z)
$$

depending on the model, are written as 


$$
\begin{aligned}
& \Psi_{\mathrm{L}, \mathrm{D}}^{(c)}(z)=\sum_{p=1}^{2 N+1} \Psi_{\mathrm{L}, \mathrm{D}}^{(c)}(z)\left[\theta\left(z-z_{p-2}\right)-\theta\left(z-z_{p-1}\right)\right], \quad z_{-1} \rightarrow-\infty, \quad z_{2 N} \rightarrow \infty, \\
& \Psi_{\mathrm{L}, \mathrm{D}}^{(o)}(z, E)=\sum_{p=0}^{2(N+1)} \Psi_{L, D(p)}^{(o)}(z, E)\left[\theta\left(z-z_{p-2}\right)-\theta\left(z-z_{p-1}\right)\right], \quad z_{-2} \rightarrow-\infty, z_{-1}=0, z_{2 N+1} \rightarrow \infty,
\end{aligned}
$$

where,

$$
\begin{aligned}
& \Psi_{D(p)}^{(\mathrm{c}, \mathrm{o})}(z)=A_{D(p)}^{(\mathrm{c}, \mathrm{o})} e^{i K_{p}(E) z}+B_{D(p)}^{(\mathrm{c}, \mathrm{o})} e^{-i K_{p}(E) z}, \\
& K_{p}(E)=\left\{\begin{array}{l}
k=\hbar^{-1} \sqrt{2 m_{w} E}, \quad \text { in well } \\
\chi=-\hbar^{-1} \sqrt{2 m_{b}(E-U)}, \quad \text { in barrier }
\end{array}\right. \\
& \Psi_{L,\left(\begin{array}{l}
1 \\
0
\end{array}\right)}^{(\mathrm{c}, \mathrm{o})}(z, E)=A_{L,\left(\begin{array}{l}
1 \\
0
\end{array}\right)}^{(\mathrm{c}, \mathrm{o})} e^{i k z}+B_{L,\left(\begin{array}{l}
1 \\
0
\end{array}\right)}^{(\mathrm{c}, \mathrm{o})} e^{-i k z}, \quad z \leq\left(\begin{array}{c}
z_{0} \\
z_{-1}
\end{array}\right) \\
& \Psi_{L,(p)}^{(\mathrm{c}, \mathrm{o})}(z, E)=A_{L,(p)}^{(\mathrm{c}, \mathrm{o})} \operatorname{Ai}[\xi(z)]+B_{L,(p)}^{(\mathrm{c}, \mathrm{o})} \operatorname{Bi}[\xi(z)], \quad(p)=\left(\begin{array}{c}
1 \\
0
\end{array}\right), \ldots,\left(\begin{array}{c}
2 N+1 \\
2(N+1)
\end{array}\right) \\
& \Psi_{L,(S)}^{(\mathrm{c}, \mathrm{o})}(z, E)=A_{L,(S)}^{(\mathrm{c}, \mathrm{o})} e^{i K_{s}(E+e F d) z}, \quad z \geq\left(\begin{array}{c}
z_{2 N+1} \\
z_{2(N+1)}
\end{array}\right), \quad S=\left(\begin{array}{c}
z_{2 N+1} \\
z_{2(N+1)}
\end{array}\right) \\
& k=\hbar^{-1} \sqrt{2 m_{w} E}, \quad \xi(z)=-\left[\frac{2 m(z)}{(\hbar e F)^{2}}\right]^{1 / 3}(U(z)-E-e F z) .
\end{aligned}
$$

The fitting conditions

$$
\left.\begin{array}{c}
\Psi_{L, D(p)}^{(\mathrm{c}, \mathrm{o})}\left(z_{p}\right)=\Psi_{L, D(p+1)}^{(\mathrm{c}, \mathrm{o})}\left(z_{p}\right) \\
\left.\frac{d \Psi_{L, D(p)}^{(\mathrm{c}, \mathrm{o})}(z)}{m_{p} d z}\right|_{z=z_{p}}=\left.\frac{d \Psi_{L, D(p+1)}^{(\mathrm{c}, \mathrm{o})}(z)}{m_{p+1} d z}\right|_{z=z_{p}}
\end{array}\right\}
$$

and normality ones

$$
\int_{-\infty}^{\infty} \Psi_{L, D ; n}^{(c) *}(z) \Psi_{L, D ; n^{\prime}}^{(c)}(z) d z=\delta_{n n^{\prime}}, \quad \int_{-\infty}^{\infty} \Psi_{\mathrm{L}, \mathrm{D}}^{(o) *}(z, k) \Psi_{\mathrm{L}, \mathrm{D}}^{(o)}\left(z, k^{\prime}\right) d z=\delta\left(k-k^{\prime}\right)
$$

completely define the electron wave functions in both models $\left(\Psi_{L, D ; n}^{(c)}(z)\right.$ and $\left.\Psi_{\mathrm{L}, \mathrm{D}}^{(o)}(z, k)\right)$ and the stationary energy spectrum $\left(E_{n}\right)$ with oscillator strengths of quantum transitions in the closed one

$$
f_{n n^{\prime}}=2\left(E_{n}-E_{n^{\prime}}\right) m \hbar^{2}\left|\int_{-\infty}^{\infty} \Psi_{L, D ; n}^{(c) *}(z) z \Psi_{L, D ; n^{\prime}}^{(c)}(z) d z\right|^{2}, \quad\left(\frac{1}{m}=\frac{P_{w}}{m_{w}}+\frac{P_{b}}{m_{b}}\right)
$$

where $P_{w}, P_{b}$ are the probabilities of electron location in the well (w) and barrier (b), respectively.

The resonance energies (RE) $E_{n}$ and resonance widths (RW) $\Gamma_{n}$ of the electron QSS in the open model can be defined within three different approaches [41], Fig 2, depending on the convenience of calculations. Using the known wave functions $\Psi_{\mathrm{L}, \mathrm{D}}^{(o)}(z, E)$, one can obtain the densities of probability distribution functions of electron location in nanostructure

$$
W_{\mathrm{L}, \mathrm{D}}^{(o)}(E)=d^{-1} \int_{0}^{d}\left|\Psi_{\mathrm{L}, \mathrm{D}}^{(o)}(z, E)\right|^{2} d z,
$$

or calculate the densities of input $j_{\text {in }}(E)$ and output 
$j_{\text {out }}(E)$ currents and, since, the transmitting coefficient

$$
D(E)=j_{\text {out }}(E) / j_{\text {in }}(E) .
$$

The position of the maxima of both these functions in energy scale define the $\mathrm{RE}\left(E_{n}\right)$ and widths of their peaks at the half of the height - the RW $\left(\Gamma_{n}\right)$ of the QSS. Using the known wave functions one can also obtain the $S$-matrix, which is the function of the complex energy $\widetilde{E}$. Its real and imaginary parts define the RE and RW of n-th QSS, Fig 2c,

$$
E_{n}=\operatorname{Re}\left[\operatorname{Pol}_{n} S(\widetilde{E})\right], \quad \Gamma_{n}=\operatorname{Im}\left[\operatorname{Pol}_{n} S(\widetilde{E})\right] .
$$

The basics of the theory of dynamic conductivity in open RTS $[39,40]$ are the following. It is assumed that the interaction between the electron and electromagnetic field, characterized by the intensity of electric field $\left(F_{a c}\right)$ and frequency of electromagnetic field $(\omega)$ in dipole approximation is described by the Hamiltonian

$\mathcal{H}(z, t)=-e F_{a c} \cos (\omega t)\{z[\theta(z)-\theta(z-d)]+d \theta(z-d)\}$.

The time and $z$-coordinate dependent wave function satisfies the complete Schrodinger equation

$$
i \hbar \frac{\partial \Psi_{\mathrm{L}, \mathrm{D}}^{(o)}(z, t)}{\partial t}=\left[H_{\mathrm{L}, \mathrm{D}}^{(o)}(z)+\mathcal{H}(z, t)\right] \Psi_{\mathrm{L}, \mathrm{D}}^{(o)}(z, t) .
$$

In one-mode approximation the solution of this equation is written as

$$
\begin{gathered}
\Psi_{\mathrm{L}, \mathrm{D}}^{(o)}(z, t)=\Psi_{\mathrm{L}, \mathrm{D}}^{(o)}(z) e^{i \omega_{0} t}+\Psi_{+1, L, D}^{(o)}(z) e^{i\left(\omega_{0}+\omega\right) t} \\
+\Psi_{-1, L, D}^{(o)}(z) e^{-i\left(\omega_{0}-\omega\right) t} \\
\left(\omega_{0}=\hbar^{-1} E\right)
\end{gathered}
$$

Here, the function $\Psi_{\mathrm{L}, \mathrm{D}}^{(o)}(z)$ is known from (6). The function $\Psi_{ \pm 1, L, D}^{(o)}(z)$ is analytically exactly obtained from the system of two non-homogeneous equations

$$
\begin{aligned}
{\left[H_{\mathrm{L}, \mathrm{D}}^{(o)}(z)\right.} & \left.-\hbar\left(\omega_{0} \pm \omega\right)\right] \Psi_{ \pm 1, L, D}(z, t)= \\
& -e F_{a c}\{z[\theta(z)-\theta(z-d)]+d \theta(z-d)\}
\end{aligned}
$$

Finally, the wave functions $\Psi_{\mathrm{L}, \mathrm{D}}^{(o)}(z, t)$ and Fourier-images of densities of electronic currents through the RTS are obtained as

$$
\begin{aligned}
& j_{\mathrm{L}, \mathrm{D}}(E \pm \Omega, z)=\frac{i e \hbar n_{0}}{2 m(z)}\left[\Psi_{ \pm 1, L, D}(E, z) \frac{\partial}{\partial z} \Psi_{ \pm 1, L, D}^{*}(E, z)\right. \\
& \left.-\Psi_{ \pm 1, L, D}^{*}(E, z) \frac{\partial}{\partial z} \Psi_{ \pm 1, L, D}(E, z)\right], \quad(\Omega=\hbar \omega)
\end{aligned}
$$

here $n_{0}$ is the concentration of uncoupling electrons.
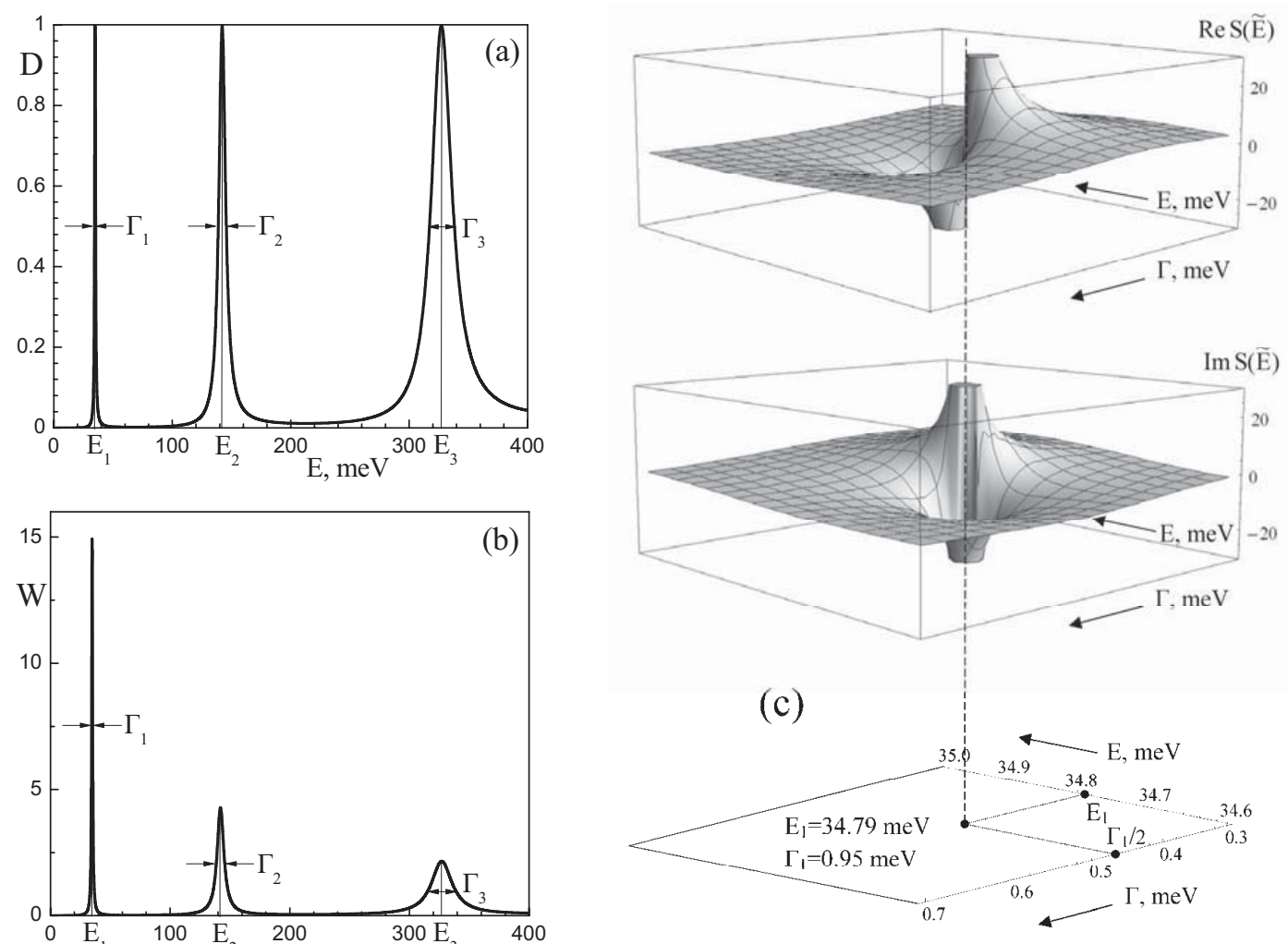

Fig. 2. Functions $W(E)(\mathrm{a}), D(E)$ (b) and $S$-matrix as function of $\widetilde{E}$ and position of its first pole in the complex plane of energy (c). 
The calculation of energetic balance between the input and output electronic currents fixes the dynamic conductivity in quasi-classic approximation

$$
\sigma_{\mathrm{L}, \mathrm{D}}=\sigma_{\mathrm{L}, \mathrm{D}}^{+}+\sigma_{\mathrm{L}, \mathrm{D}}^{-}
$$

where its partial terms

$$
\begin{aligned}
\sigma_{\mathrm{L}, \mathrm{D}}^{ \pm} & = \pm \Omega\left(2 e d F_{a c}^{2}\right)^{-1}\left(j_{\mathrm{L}, \mathrm{D}}\left[E+\Omega,\left(\begin{array}{l}
d \\
0
\end{array}\right)\right]\right. \\
& \left.-j_{\mathrm{L}, \mathrm{D}}\left[E-\Omega,\left(\begin{array}{l}
d \\
0
\end{array}\right)\right]\right),
\end{aligned}
$$

describe the input $\left(\sigma_{\mathrm{L}, \mathrm{D}}^{+}\right)$and output $\left(\sigma_{\mathrm{L}, \mathrm{D}}^{-}\right)$currents through the RTS, respectively.

\section{CALCULATION AND ANALYSIS OF THE DESIGN OF THREE-BARRIER RTS AS AN ACTIVE REGION OF THE CASCADES OF QCD AND QCL IN CLOSED AND OPEN MODELS}

The theory developed in the previous Section for the two models of multilayered RTS gives an opportunity to calculate the design of QCD and QCL cascades and reveals the optimal configurations of their active regions, which provide the functioning of these nano-devices at the needed frequencies of the electromagnetic field.

We calculated the design of QCD and QCL cascades for the RTS with the same compositions and number of layers. For the sake of comparison with the experimental data we studied the RTS composed of $\operatorname{In}_{0.53} \mathrm{Ga}_{0.47} \mathrm{As}-$ wells and $\mathrm{In}_{0.52} \mathrm{Al}_{0.48}$ As-barriers. They were realized as the cascades of these nano-devices in the papers [42, 43]. The computer calculations were performed using the physical parameters $m_{w}=0.045 m_{0}, m_{b}=0.086 m_{0}$, $U=516 \mathrm{meV}, n_{0 D}=4 \cdot 10^{17} \mathrm{~cm}^{-3}, n_{0 L}=2 \cdot 10^{17} \mathrm{~cm}^{-3}$, $F=68 \mathrm{kV} / \mathrm{cm}$. The sizes of the cascades elements (active region, extractor, injector) and that of extractor wells and barriers were taken the same as in the cited papers because they are easily and rather exactly calculated in the simple model of "uncoupling wells", in order to create the system of energy levels producing the "phonon ladder" in the QCD cascade.

As an example, we studied the multilayered RTS (Fig. 1,b), as a separate cascade of QCD.The configuration of RTS, being the active region, is varying due to the different location of the inner barrier between the outer ones, that means the varying size of the input well $\left(b_{1}\right)$ at such fixed widths of both wells of active region which correspond to the experiment [42]. In Fig. 3 the energy spectrum and oscillator strengths $f_{1 n}$ in the model of closed cascade, resonance energies $E_{n}$ with logarithms of life times $\ln \tau_{n}\left(\tau_{n}=\hbar \Gamma^{-1}\right.$ in units $\left.\tau_{0}=1 \mathrm{ps}\right)$ of electrons in both operating QSS and logarithms of conductivities $\ln \sigma_{12}, \ln \sigma_{12}^{ \pm}, \ln \sigma_{13}$ (in the units $\sigma_{0}=1 \mathrm{~S} / \mathrm{cm}$ ) in a model of open cascade are presented as functions of the width of the input well $\left(b_{1}\right)$ in a three-barrier active region at fixed geometrical parameters of other cascade elements. It is clear that the dependences of electron spectrum $E_{n}$ on $b_{1}$ in closed and open models are the same with the exactness not less than 0.1 percent.
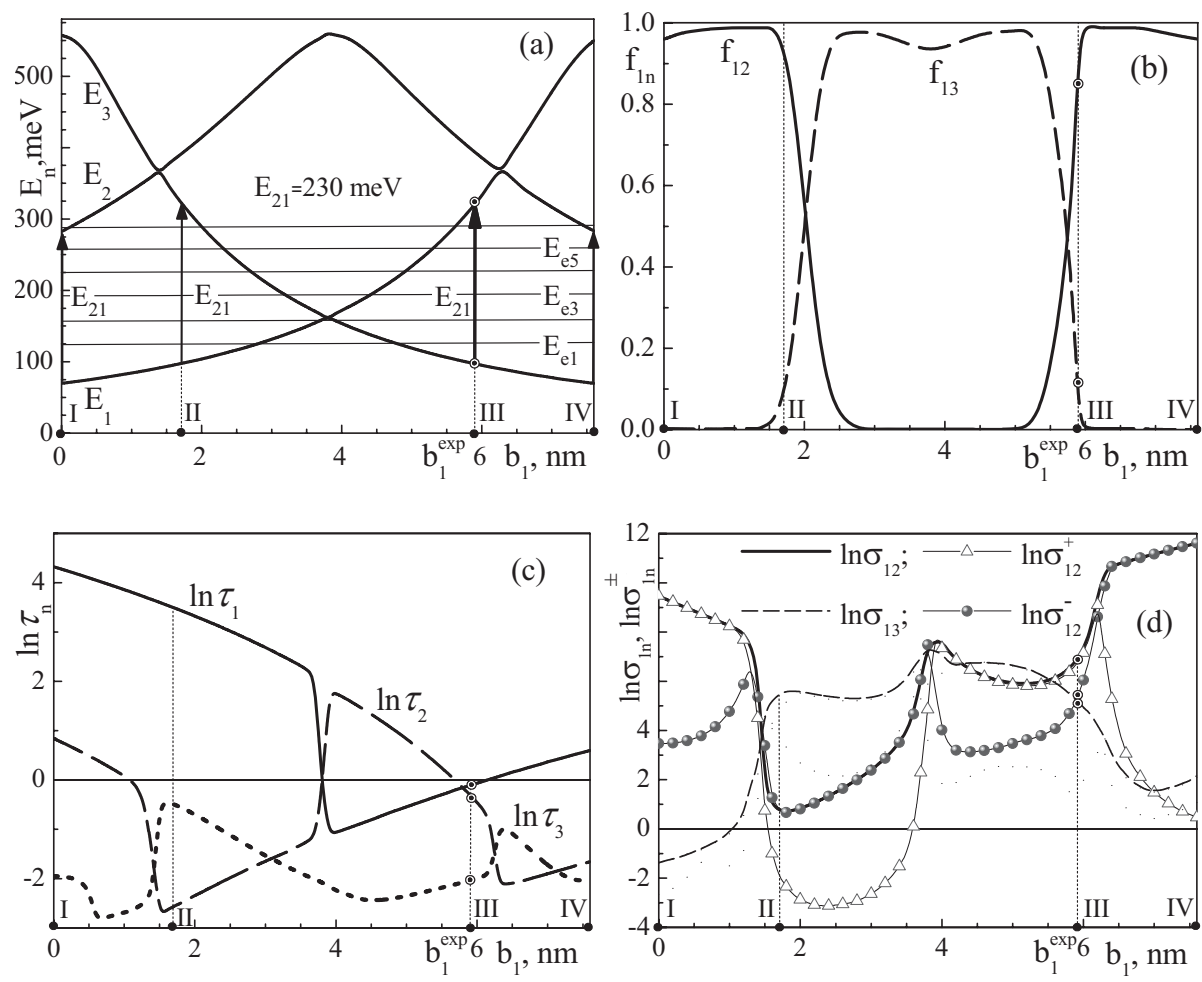

Fig. 3. Energies $E_{n}, E_{\text {en }}$ (a), oscillator strengths $f_{12}, f_{13}$ (b) in closed model; resonance energies $E_{n}, E_{\text {en }}$ (a), logarithms of life times $\tau_{n}$ (c), logarithms of conductivities $\sigma_{12}, \sigma_{13}$ and partial terms $\sigma_{12}^{ \pm}$in open model (d) as functions of the input well width $b_{1}$. 
Fig. 3,a proves that RE $E_{n}$ (bold curves) of operating states in which the electron is mainly located in the active region, non-monotonously depend on $b_{1}$ displaying the anti-crossing effect which is typical for the RTS. The RE $E_{\text {en }}$ (thin curves) of QSS in which the electron with bigger probability is in a cascade extractor almost do not depend on the design of active region and are determined only by geometrical parameters of the extractor. The distances between $E_{e n}$ and $E_{e(n-1)}$ in the experimental QCD are of the order of optical phonon energy $\Omega_{\mathrm{ph}}=32-34 \mathrm{meV}$. Hence, the electrons relaxing at the phonon subsystem perform a radiationless quantum transition from the $|2\rangle$ operating QSS into $|1\rangle$ QSS of the next QCD cascade.

In the closed model, the QCD which detects the electromagnetic field with the energy $E_{21}=E_{2}-E_{1}$ as a result of a quantum transition $|1\rangle \rightarrow|2\rangle$ optimally operates when the oscillator strength $f_{12}$ is maximal at $f_{12}>f_{1(n>3)}$. Fig. 3a,b prove that this condition fulfills for the four different geometrical configurations of the active region, none of which has definite advantages over the other.

For the coordinated operation of all QCD cascades in the model of open RTS it is necessary that conductivity $\left(\sigma_{12}\right)$ in quantum transition accompanied by the absorption of energy $E_{21}$ would be maximal, much bigger than the conductivities $\left(\sigma_{1(n>3)}\right)$ produced by other transitions. Besides, its partial term $\left(\sigma_{12}^{+}\right)$in the forward current through the RTS into the next cascade would be much bigger than that $\left(\sigma_{12}^{-}\right)$of the backward current, namely $\sigma_{12}^{+} \gg \sigma_{12}^{-}$. Fig. 3c,d show that not all ge- ometrical configurations of the active region, which produce energy $E_{21}$, are optimal for the QCD operation. So in the first (I) configuration the conditions $\sigma_{12}>\sigma_{13}$, $\sigma_{12}^{+}>\sigma_{12}^{-}$are fulfilled but the life time of the electron in the ground QSS is essential $\left(\tau_{1}>10 \mathrm{ps}\right)$, which leads to the accumulation of harmful dynamic charge in the system. In the second (II) configuration, the conductivity produced by the quantum transition $|1\rangle \rightarrow|3\rangle$ prevails, since, $\sigma_{13}>\sigma_{12}$. The fourth (IV) configuration is also not effective for the QCD operation because here $\sigma_{12}^{-} \gg \sigma_{12}^{+}$.

Thus, there is only one third (III) configuration with the narrow region $\left(5.6 \mathrm{~nm} \leq b_{1} \leq 6.2 \mathrm{~nm}\right)$ where to place the inner barrier between the outer ones in an active region, herein QCD would operate in the optimal regime because $\sigma_{12}^{+} \gg \sigma_{12}^{-} ; \tau_{1}, \tau_{2}<3$ ps. We should note that in the experimentally realized design of the QCD active region, the inner barrier is placed just in this region $b_{1}^{\exp }=5.9 \mathrm{~nm}$. The calculation proves that the theoretically obtained energies of the detected field $E_{21}=230$ meV differ from the experimental one $E_{21}^{\exp }=232.2 \mathrm{meV}$ less than by 1 percent and the difference of energies of electron states localized in the extractor is close to the energy of optical phonons.

In the same way as for the above-described detector, we calculated the physical parameters $\left(E_{n}, E_{i n}, f_{n n^{\prime}}\right.$, $\left.\ln \tau_{n}, \ln \sigma_{n n^{\prime}}\right)$ in both models for the QCL cascade, realized in paper [43], driven by the constant electric field, Fig. 1,a. In laser quantum transitions from upper into the lower QSS, the negative dynamic conductivity appears as it must be.
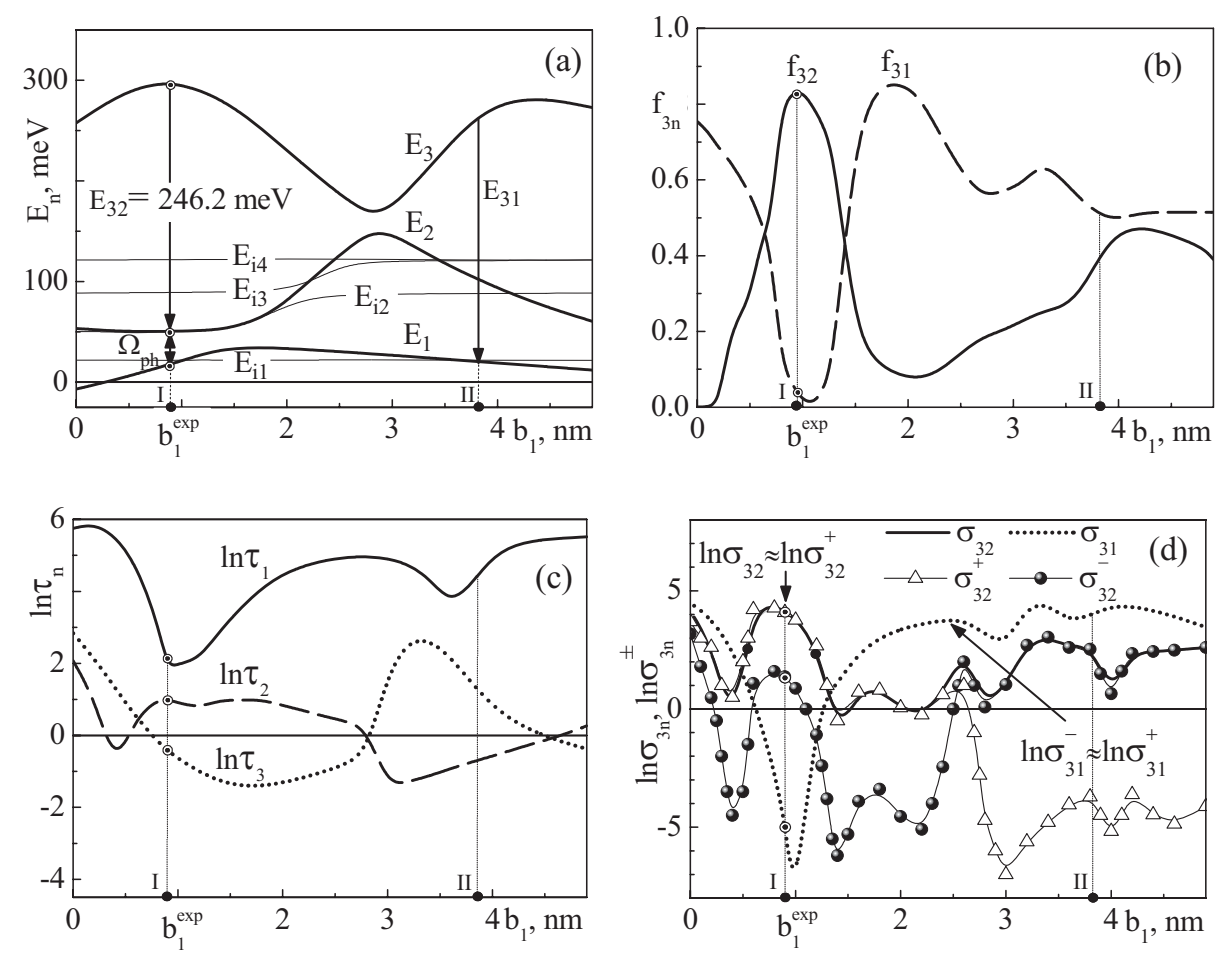

Fig. 4. Energies $E_{n}, E_{i n}$ (a), oscillator strengths $f_{12}, f_{13}$ (b) in closed model; resonance energies $E_{n}, E_{i n}$ (a), logarithms of life times $\tau_{n}$ (c), logarithms of conductivities $\sigma_{32}, \sigma_{31}$ and partial terms $\sigma_{32}^{ \pm}$in open model (d) as functions of the input well width $b_{1}$. 
The results of the calculated dependences of the energies $\left(E_{n}, E_{i n}\right)$, oscillator strength $\left(f_{n n^{\prime}}\right)$, life time $\left(\ln \tau_{n}\right)$ and dynamic conductivity $\left(\ln \sigma_{n n^{\prime}}\right)$ on the configuration of three-barrier RTS, determined by the width of the input well $\left(b_{1}\right)$ are shown in fig. 4 . It proves that all three energies of operating levels $\left(E_{1}, E_{2}, E_{3}\right)$ are equal in both models and essentially depend on $b_{1}$ while injector energy levels $\left(E_{i n}\right)$ almost do not depend on it. It is clear that both models contain two configurations (I, II), the quantum transitions in which $(|3\rangle \rightarrow|2\rangle$ and $|3\rangle \rightarrow|1\rangle)$ can provide radiation of the electromagnetic field with the wanted energy $\sim 246 \mathrm{meV}$ (Fig. 4a).

Herein, the analysis of the oscillator strengths in the closed model (Fig. 4b) does not answer the question: why at the comparable $f_{32}^{(\mathrm{I})} \approx 0.83$ and $f_{31}^{(\mathrm{II})} \approx 0.52$, the QCL cannot operate in II-configuration while in the I-one, as experiment [43] proves, it operates successfully. On the contrary, the open model clearly answers this question. Indeed, from Figs. 4,c,d it is clear that in IIconfiguration the life times in $|3\rangle$ and $|1\rangle$ QSS are very big and, that the main ???, $\sigma_{31} \sim \sigma_{31}^{-}$as far as $\sigma_{31}^{-} \gg \sigma_{13}^{+}$. Thus, the dynamic conductivity is formed by the backward current of electrons over the cascade, consequently, the electrons from the $|1\rangle$ and $|2\rangle$ QSS of the previous cascade do not get into the $|3\rangle$ QSS of the next one, since the laser does not operate. The I-configuration corresponds to the experimental design of cascade [43] with the three-level scheme of QCL. The same figure proves that the life times in $|3\rangle$ and $|2\rangle$ operating QSS are comparable and rather small, which causes their rapid release of electrons (the radiationless transition $|2\rangle \rightarrow|1\rangle$ accompanied by the creation o af phonon also contributes to this). It is very important that in this configuration conductivity $\sigma_{32}$ has the maximum but it is much bigger than $\sigma_{31}\left(\sigma_{32} \gg \sigma_{31}\right)$ and $\sigma_{32}^{+} \gg \sigma_{32}^{-}$. Hence, it is clear that conductivity $\sigma_{32}$ is produced by the forward main electronic current from $|2\rangle$ and $|1\rangle$ QSS of the previous cascade at $|3\rangle$ QSS of the next one. At these conditions the operation of laser cascades is well coordinated and QCL functions successfully. We should also note that in the I-configuration the theoretical radiation energy $E_{32}=246.2 \mathrm{meV}$ to within $3 \%$ coincides with the experiment [43] and the difference of energies $E_{2}-E_{1} \approx 34 \mathrm{meV}$ correlates well with the energy of polarization phonon of crystals which form a quantum well.

\section{ELECTRON-PHONON INTERACTION IN RTS}

As was mentioned above, phonons play an important role for the functioning of QCD and QCL. On the one hand, it is a destructive sub-system the interaction with which, for example, destroys the coherence of electron quasi-stationary states in different cascades of QCL. On the other hand, their positive feature is that this interaction creates the mechanism of radiationless transmission of electronic energy from the excited into ground QSSs for the effective operation of QCL and QCD. The purpose of the theory of electron-phonon interaction in plane semiconductor nano-heterostructures is to obtain the energy spectra and potentials of polarization fields in order to study different problems, for example, the renormalized electron spectrum and radiationless quantum transitions between electron QSSs.

Let us observe the plane nano-heterostructure consisting of $N$ semiconductor layers embedded into the outer medium. Each of them is characterized by a dielectric constant which is given by the Lidden-Sacks-Teller expression for a binary composition and for a ternary one [44]:

$$
\begin{aligned}
\varepsilon_{j}(\Omega) & =\varepsilon_{\infty j} \frac{\Omega^{2}-\Omega_{L j}^{2}}{\Omega^{2}-\Omega_{T j}^{2}} \\
\varepsilon_{j}(\Omega) & =\varepsilon_{\infty j} \frac{\left(\Omega^{2}-\Omega_{L j 1}^{2}\right)\left(\Omega^{2}-\Omega_{L j 2}^{2}\right)}{\left(\Omega^{2}-\Omega_{T j 1}^{2}\right)\left(\Omega^{2}-\Omega_{T j 2}^{2}\right)} .
\end{aligned}
$$

The high frequency dielectric constant $\varepsilon_{\infty}$ and energies (or frequencies) of longitudinal (L) or transversal (T) phonons are known from the experiments for the respective bulk crystals.

Combining the system of Maxwell equations for the potential of polarization field of RTS in the dielectric continuum model, the equation $\varepsilon(\omega) \Delta \Phi(\mathbf{r})=0, \omega=\Omega / \hbar$ is obtained $[45,46]$. Due to invariantness in the plane $x o y$, $\Phi(\mathbf{r})=\sum_{\mathbf{q}} \Phi(z) \exp (i \mathbf{q} \rho)$, it is transformed to the equation

$$
\varepsilon(\omega)\left(\frac{\partial^{2}}{\partial z^{2}}-q^{2}\right) \Phi(z)=0 .
$$

At the conditions

$$
\varepsilon_{j}(\omega)=0, \quad \Delta \Phi_{L}^{(j)}(\mathbf{r}) \neq 0, \quad \Phi_{L}^{(j)}\left(z_{j}\right)=0
$$

we obtain the spectrum and potential of polarization field of confined (L) phonons, while at

$$
\varepsilon(\omega) \neq 0, \quad \Delta \Phi_{I}(\mathbf{r})=0
$$

- that of interface (I) phonons.

From formulas (23), (25) one can see that the spectrum of confined phonons contain the same dispersionless longitudinal phonons as the respective bulk binary $\left(\Omega_{L j}=\hbar \omega_{L j}\right)$ or ternary $\left(\Omega_{L j l}=\hbar \omega_{L j l}, l=1,2\right)$ crystals. The potential of their polarization field is defined by the formula

$$
\Phi_{L}^{(j)}=\sum_{\mathbf{q}}\left[A_{j q} \sin (q z)+B_{j q} \cos (q z)\right],
$$

where the unknown coefficients are obtained from fitting conditions.

The potential of polarization field of I-phonons is found from equations (26). Their solutions are fixed by the formulas

$$
\begin{aligned}
& \Phi_{I}=\sum_{j=0}^{N+1} \Phi_{I}^{(j)}(q, z)\left[\theta\left(z-z_{j}\right)-\theta\left(z-z_{j-1}\right)\right], \\
& \Phi_{I}^{(j)}(q, z)=\alpha_{j} e^{-q z}+\beta_{j} e^{q z}
\end{aligned}
$$


with fitting conditions

$$
\begin{array}{r}
\Phi_{I}^{(j)}\left(q, z_{j}\right)=\Phi_{I}^{(j+1)}\left(q, z_{j}\right) ; \\
\left.\varepsilon^{(j)}(\Omega) \frac{\partial \Phi_{I}^{(j)}(q, z)}{\partial z}\right|_{z=z_{j}} \\
=\left.\varepsilon^{(j+1)}(\Omega) \frac{\partial \Phi_{I}^{(j+1)}(q, z)}{\partial z}\right|_{z=z_{j}}
\end{array}
$$

The latter are solved using the transfer-matrix method [44], since the energy spectrum of I-phonons is given by the equation

$$
\operatorname{det}[T(\Omega, q)-I]=0,
$$

where $I, T$ are the identity and transfer matrices, respectively.

For the investigation of electron spectrum renormalized due to the interaction with phonons using the method of Green's functions, the Hamiltonian of electron-phonon system is written in the representation of the second quantization over all variables $[45,46]$

$$
H=H_{e}+H_{L}+H_{I}+H_{e-L}+H_{e-I},
$$

where

$$
H_{e}=\sum_{n \mathbf{k}} E_{n \mathbf{k}} a_{n \mathbf{k}}^{+} a_{n \mathbf{k}}
$$

is the electron Hamiltonian,

$$
\begin{aligned}
H_{L} & =\sum_{j} \sum_{l}^{2} \sum_{\lambda \mathbf{q}} \Omega_{j l}\left(b_{j l \lambda \mathbf{q}}^{+} b_{j l \lambda \mathbf{q}}+1 / 2\right), \\
H_{I} & =\sum_{s, \mathbf{q}} \Omega_{s \mathbf{q}}\left(b_{s \mathbf{q}}^{+} b_{s \mathbf{q}}+1 / 2\right)
\end{aligned}
$$

is the Hamiltonians of L- and I-phonons,

$$
\begin{gathered}
H_{e-L}=\sum_{n_{1} n j \mathbf{k} l \lambda \mathbf{q}} F_{n_{1} n}^{j l}(\lambda, q) a_{n_{1} \mathbf{k}+\mathbf{q}}^{+} a_{n \mathbf{k}}\left(b_{j l \lambda \mathbf{q}}+b_{j l \lambda,-\mathbf{q}}^{+}\right),(34) \\
H_{e-I}=\sum_{n_{1} n \mathbf{k}} \sum_{s \mathbf{q}} F_{n_{1} n \mathbf{k}}(s, q) a_{n_{1} \mathbf{k}+\mathbf{q}}^{+} a_{n \mathbf{k}}\left(b_{s, \mathbf{q}}+b_{s,-\mathbf{q}}^{+}\right),
\end{gathered}
$$

is the Hamiltonians of $\mathrm{e}-\mathrm{L}-$ and $\mathrm{e}-\mathrm{I}$ interaction. $F_{n_{n}^{\prime} n}^{j l}(\lambda, q), F_{n_{1} n \mathbf{k}}(s, q)$ are the e-L- and e-I binding functions.

The Hamiltonian of ann electron-phonon system in RTS (31) is definitely obtained from the first principles without any fitting parameters. At small concentrations of electrons and their weak binding with phonons, the Fourier-image of electron Green's function is found from Dyson equation [47]:

$$
G_{n}(\mathbf{k}, \hbar \omega)=\left[\hbar \omega-E_{n \mathbf{k}}-M_{n}(\hbar \omega, \mathbf{k})\right]^{-1}
$$

with the mass operator $M_{n}(\hbar \omega, \mathbf{k})$ calculated in onephonon approximation

$$
M_{n}(\hbar \omega, \mathbf{k})=\sum_{n_{1} p \mathbf{q}} F_{n n_{1}}^{*}(p, q) F_{n_{1} n}(p, q)\left[\frac{1+\nu_{p \mathbf{q}}}{\hbar \omega-E_{n_{1}}(\mathbf{k}-\mathbf{q})-\Omega_{p \mathbf{q}}+i \eta}+\frac{\nu_{p \mathbf{q}}}{\hbar \omega-E_{n_{1}}(\mathbf{k}+\mathbf{q})+\Omega_{p \mathbf{q}}+i \eta}\right]
$$
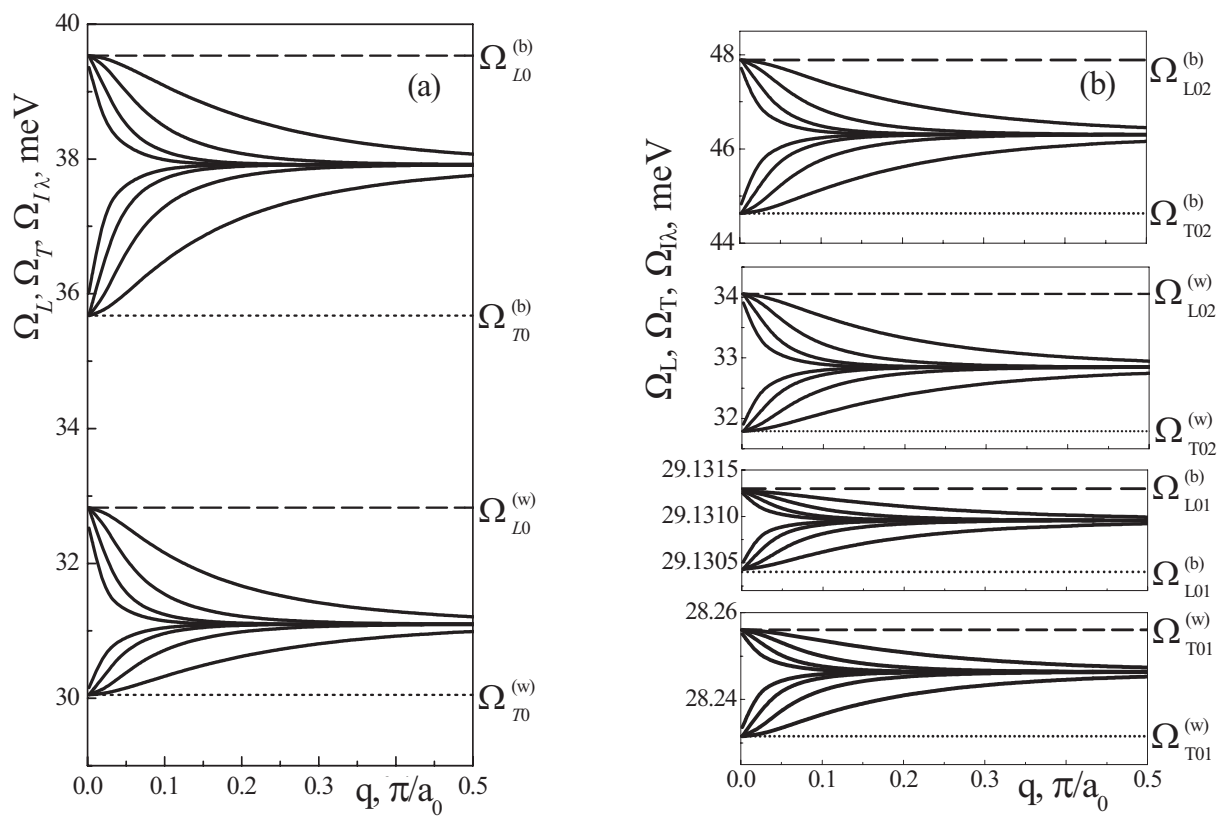

Fig. 5. Spectrum of confined L- and I-phonons in RTS, being the active region of QCD in binary- (a) and ternary compound (b) models. 
The first term of mass operator describes the processes of electron-phonon interaction accompanied by the creation of phonons with an average occupation number $\nu_{p \mathbf{q}}=\left(e^{\Omega_{p \mathbf{q}} / \mathrm{k}_{B} T}-1\right)^{-1}$ and the second one by annihilation. Here the generalized index for the phonon modes $(p)$ a numerates all L-phonons modes at $p=j, \lambda$ and all I-phonon modes at $p=s$.

Considering that the electrons move perpendicularly to the planes of RTS, with the energy close to the energy of the bottom of respective electronic band, we put $\mathbf{k}=0$ in (37) and neglect the frequency dependence of mass operator in the vicinity of electron energies $E_{n}$ taking into account a weak electron-phonon binding. Now, the real and imaginary parts of mass operator determine the shift $\left(\Delta_{n}=\operatorname{Re} M_{n}\left(\hbar \omega=E_{n}\right)\right)$ and decay rate $\left(\Gamma_{n}=-\operatorname{Im} M_{n}\left(\hbar \omega=E_{n}\right)\right)$ of the $n$-th electronic state.

The developed theory is used for the calculation of phonon spectra and parameters of renormalized electron spectrum for the closed three barrier RTS $\left(\mathrm{GaAs} / \mathrm{Al}_{0.34} \mathrm{Ga}_{0.66} \mathrm{As}\right)$, being the active region of QCD studied in paper [7]. The spectra of L- and I-phonons in binary and ternary compound models are presented in figure 5. It is seen that in the binary model there are two energies of confined L-phonons and two groups containing 8 modes of I-phonons each, with weak dispersion $(3-4 \mathrm{meV})$. In the ternary model there are four energies of confined L-phonons and sixteen groups containing 8 modes of I-phonons each, also with weak dispersion. Herein, two lower groups of energies are almost dispersionless and upper two have the same dispersion as their analogues in the binary model. In each group of Iphonons the number of modes is equal to twice the number of interfaces in RTS. This obstacle can be very important to the effective operation of the QCD extractor "smoothing" the experimental deviations in the phonon ladder of electron QSSs.

The energy shifts $\left(\Delta_{12}^{T}=\Delta_{2}^{T}-\Delta_{1}^{T}\right)$ and widening $\left(\Gamma_{12}^{T}=\Gamma_{1}^{T}+\Gamma_{2}^{T}\right)$ of absorption band of electromagnetic field in the transition of electron from state $|1\rangle$ into state $|2\rangle$ due to the electron-phonon interaction in three barrier RTS depending on its configuration are shown in figure 6 at $T=300 \mathrm{~K}$. It is seen that the shift of the band can vary its sign but its magnitude is small (approximately $1 \mathrm{meV}$ ). The width of the band is also small and varies in the range $1-2 \mathrm{meV}$. In the configuration of the experimental active region of $\mathrm{QCD}$, the electronphonon interaction shifts the absorption band into the high-energy region at $0.5 \mathrm{meV}$ widening it at $1.5 \mathrm{meV}$ when the temperature varies from the cryogenic to the room one. At the same time, due to a smaller height of RTS potential barriers, the difference between the energies of operating QSS decreases as compared with $E_{12}=E_{2}-E_{1}$ at the increasing temperature. It causes the shift of absorption band into the low-energy region at the magnitude of $4.2 \mathrm{meV}$. The additional broadening of this band happens because the RTS is an open one due to the broadening of the bands of operating electron QSSs. As a result, theoretical magnitudes of the shifts and broadening of the absorption band of electromagnetic field correlate well with the experimental data.

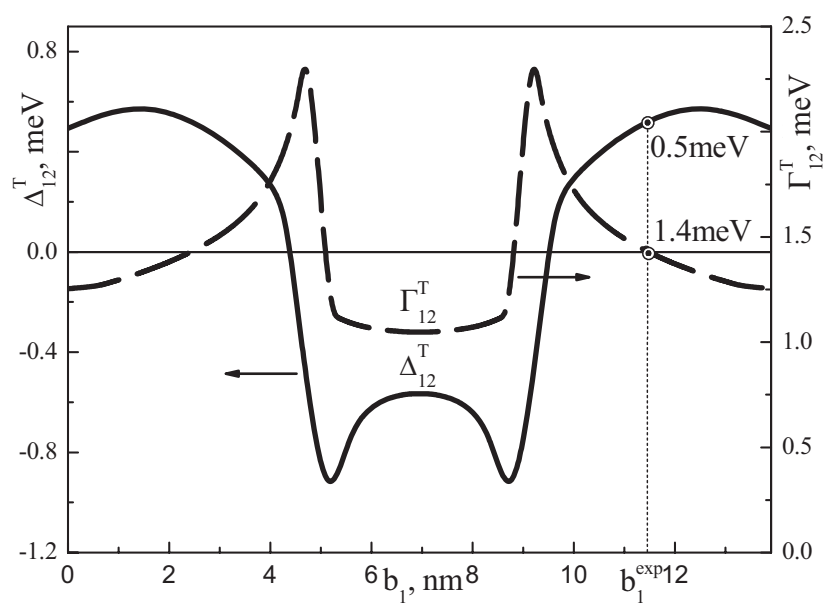

Fig. 6. Dependence of temperature shift $\Delta_{12}^{T}$ and broadening $\Gamma_{12}^{T}$ of absorption band of electromagnetic field on geometrical design of RTS at $T=300 \mathrm{~K}$.

\section{CONCLUSION}

The theory of electronic dynamic conductivity and spectral parameters (resonance energies and widths) of their quasi-stationary states is established within the effective mass approximation and models of rectangular and triangular potential profiles of the resonant tunneling structure, respectively.

The developed quantum theory of photon- and phonon-assisted tunneling of electrons through the multilayered RTS in an open model for the cascades of QCD and QCL describes the physical processes clearly and more adequately than a closed model. Using it, the spectral parameters of absorption band (QCD) or radiation (QCL) of electromagnetic field are satisfactorily defined, with respect to the experimental data taking into account the dissipative mechanisms of interaction with phonons. The presented theory can be used to optimize the multilayered RTS parameters and to find a corresponding design of devices.
[1] R. Kazarinov, R. Suris, Sov. Phys. Semicond. 5, 797 (1971).

[2] R. Kazarinov, R. Suris, Sov. Phys. Semicond. 6, 148 (1972).

[3] B. F. Levine, K. K. Choi, C. G. Bethea, J. Walker,
R. J. Malik, Appl. Phys. Lett. 50, 1092 (1987).

[4] H. Schneider, P. Koidl, F. Fuchs, B. Dischler, K. Schwarz, J. D. Ralston, Semicond.Sci.Techn. 6, 120 (1991).

[5] J. Faist, F. Capasso, D. L. Sivco, C. Sirtori, A. L. Hutchinson, A.Y. Cho, Science 264, 533 (1994). 
[6] J. Faist, F. Capasso, C. Sirtori, D. L. Sivco, A.L. Hutchinson, A.Y. Cho, Appl. Phys.Lett. 66, 538 (1995).

[7] L.Gendron, M.Carras, A.Huynh, V. Ortiz, C. Koeniguer, V. Berger., Appl. Phys. Lett., 85, 2824 (2004).

[8] F. R. Giorgetta et al., Appl. Phys. Lett. 91, 111115 (2007).

[9] W. Lei and C. Jagadish, J. Appl. Phys. 104, 091101 (2008).

[10] C. Gmachl, F. Capasso, D.L. Sivco, A.Y. Cho, Rep.Prog.Phys. 64, 1601 (2001).

[11] P. Gutowski et al., Photonics Lett. Pol. 6(4), 142 (2014).

[12] D. Chastanet et al., Appl. Phys. Lett. 105, 111118 (2014).

[13] S. Sakr, E. Giraud, A. Dussaigne, M. Tchernycheva, N. Grandjean, F. H. Julie, Appl. Phys. Lett. 100, 181103, (2012).

[14] P. Reininger et al., Appl. Phys.Lett. 105, 091108 (2014).

[15] M. Wanke et al., Appl. Phys. Lett. 78, 3950 (2001).

[16] S. Kumar, C.W.I. Chan, C. Qing, J.L. Reno, Appl. Phys. Lett. 95, 141110 (2009).

[17] B. Schwarz et al., Appl. Phys. Lett. 107, 071104 (2015).

[18] F. R. Giorgetta et al., IEEE J. Quant. Electron. 45, 1039 (2009).

[19] T. Ouchi, Handbook of Terahertz Technology for Imaging, Sensing and Communications, (Elsevier, 2013), p. 423.

[20] S. Katz, A. Vizbaras, R. Meyer, M.-C. Amann, J. Appl. Phys. 109, 081101 (2011).

[21] M Beeler, E Trichas, E Monroy, Semicond. Sci. Technol. 28, 074022 (2013).

[22] F. Castellano, K. Ohtani, L. Nevou, J. Faist, Appl. Phys. Lett. 102, 032103 (2013).

[23] G. G. Zegryaa, N. V. Tkach, I. V. Boiko, Yu. A. Seti, Phys. Solid State 55, 2182 (2013).

[24] S.-C. Lee, F. Banit, M. Woerner, A. Wacker, Phys. Rev. B 73, 245320 (2016).

[25] M. Bugajski et al., Phys. Stat. Sol. B 251, 1144 (2014).

[26] A. Kolek, G. Haldaś, M. Bugajski, K. Pierściśski, P. Gutowski, IEEE J. Select. Topics Quant. Electron 21, 1200110 (2015).

[27] X. Gao, M. D'Souza, D. Botez, I. Knezevic, Opt. Quant. Electron. 40, 331 (2008).
[28] H Li, J C Cao, H C Liu, Semicond. Sci. Technol. 23, 125040 (2008).

[29] A. Jiang, A. Matyas, K. Vijayraghavan, C. Jirauschek, Z. R. Wasilewski, M. A. Belkin, J.Appl. Phys. 115, 163103 (2014).

[30] R. Terazzi and J. Faist, New J. Phys. 12, 033045 (2010).

[31] A. Bismuto, R. Terazzi, B. Hinkov, M. Beck, J. Faist, Appl. Phys. Lett. 12, 021103 (2012).

[32] J. Faist, Quantum Cascade Lasers (Oxford UniversityPress, Oxford, 2013).

[33] Van-Nam Do, Adv. Nat. Sci.: Nanosci. Nanotechnol. 5, 033001 (2014).

[34] C. Jirauschek, T. Kubis, Appl. Phys. Rev. 1, 011307 (2014).

[35] G. Platero, R. Aguado, Phys. Rep. 395, 1 (2004).

[36] S. Kohler, J. Lehmann, P. Hunggi, Phys. Rep. 406, 379 (2005).

[37] N. V. Tkach, Yu. A. Seti, JETP Lett. 95, 271 (2012).

[38] M. V. Tkach, Ju. O. Seti, O. M. Voitsekhivska, Acta Phys. Pol. A 124, 94 (2013).

[39] M. V. Tkach, Ju. O. Seti, I. V. Boyko, O. M.Voitsekhivska, Condens. Matt. Phys. 16, 33701 (2013).

[40] M. V. Tkach, Ju. O. Seti, I. V. Boyko, O. M. Voitsekhivska, Rom. Rep. Phys. 65, 1443 (2013).

[41] N. V. Tkach, Yu. A. Seti, Low Temp. Phys. 35, 556 (2009).

[42] D. Hofstetter, M. Graf, T. Aellen, J. Faist, L Hvozdara, S. Blaser, Appl. Phys. Let. 89, 061119 (2006).

[43] C. Sirtori, J. Faist, F. Capasso, A. Y. Cho, Pure. Appl. Opt. 7, 373 (1998).

[44] M. A. Stroscio, M. Dutta, Phonons in Nanostructures (Cambridge University Press, Cambridge, 2001).

[45] M. V. Tkach, Ju.O. Seti, Y. B. Grynyshyn, O. M. Voitsekhivska, Acta Phys. Pol. A 128, 343 (2015).

[46] Ju. Seti, M. Tkach, M. Pan'kiv, O. Voitsekhivska, Rom. Journ. Phys. 61, 980 (2016).

[47] A. A. Abrikosov, L. P. Gorkov, I. E. Dzyaloshinsky, Methods of Quantum Field Theory in Statistical Physics (Dover, New York, 2012).

\title{
ФОТОН- І ФОНОН-СУПРОВІДНИЙ ТРАНСПОРТ ЕЛЕКТРОНІВ У РЕЗОНАНСНО-ТУНЕЛЬНИХ НАНОСТРУКТУРАХ
}

\author{
М. В. Ткач, Ю. О. Сеті, О. М. Войцехівська, О. Ю. Питюк \\ Чернівецький національний університет ім. Ю. Федъковича, \\ вул. Кочюбинсъкого, 2, Чернівці, 58012, Україна
}

\begin{abstract}
Розвинено квантовомеханічну теорію фотон- і фонон-супровідного тунелювання електронів крізь відкриті багатошарові наноструктури як каскади квантових каскадних лазерів і квантових каскадних детекторів. У моделі відомих ефективних мас та прямокутного і трикутного потенціальних рельєфів резонансно-тунельної структури побудовано теорію електронної динамічної провідності та спектральних параметрів (резонансних енергї̈ та ширин) квазістаціонарних станів.

Показано, що відкрита модель багатошарових резонансно-тунельних структур, порівняно із закритою, не лише адекватно описує фізичні процеси у каскадах та їх елементах (активних зонах, екстракторах, інжекторах), а й відповідно до експерименту дозволяє без підгінних параметрів однозначно розраховувати й виявляти їх оптимальний геометричний дизайн.
\end{abstract}

